\title{
One of the Many
}

Shannon E. Scott-Vernaglia, MD

I $\mathrm{t}$ was November in New England, and the night crept in earlier each day. I was barely 2 years into my position as program director, still new and unsure of myself. The Accreditation Council for Graduate Medical Education site visit was approaching, and each evening, after a long day of seeing patients, teaching, and mentoring, I would sit at my desk and pore over the minutiae required for the dreaded Program Information Form-overwhelmed by the magnitude of responsibility I didn't know how to share. I stared out the window into darkness, past the computer screen where the day's patient notes sat unfinished. My stomach turned as I thought of the files I still had to read for applicants interviewing the next day, their eager enthusiasm unchanged even though, for me, talking to them had become a joyless chore. Transitioning home was incredibly difficult. Before I knew it, I had missed dinner and bedtime stories with my young children. One more day failed as a mother; I might as well keep working. My mind had become the darkness of the New England night, only it didn't clear when the sun rose each morning.

Blessed with an office that was my own, I would lock the door and sob, my suffering pouring out in a sea of tears. Commuting to work, I was blinded by emotion tearing through me, no longer able to listen to National Public Radio because the additional mental task was too much. I was wretched to my family if they dared to ask how I was feeling. My identity, so strongly rooted in being the one to care for others, was shattered by needing help myself. Once, red-eyed and puffy-faced, with tears streaking down my face, I ran into another faculty member at the elevator who asked if I was okay. "It's not been a great day," I replied. Who knows what she thought. I projected disinterest in talking further and she went on with her day. Readying myself to meet with residents, I would blow my nose, wash my face, and hope they wouldn't ask if something was wrongthey never did.

Depression begets isolation, and isolation begets depression. It was isolation that echoed in my head like wordless thunder. I felt unworthy of anyone's care

DOI: http://dx.doi.org/10.4300/JGME-D-19-00007.1 and concern, and it was easy to discourage any effort to show me compassion. I radiated a message of wanting to be left alone. I struggled to be present with patients and residents, to smile even though I didn't feel joy. Supporting residents in distress, I couldn't silence the drumbeat of my own anguish. I was a fraud mentoring them along a career path I had veered off with no guide of my own.

Sometimes we trust blindly that medication and therapy will cure depression in the way that antibiotics cure strep throat. When we teach about the value of antidepressants and cognitive behavioral therapy, do we unknowingly suggest that these are easy or quick solutions? In clinic, I was teaching residents how to dose the very medications that had yet to make my days any brighter; I was waking each morning checking to see if I felt suicidal. Would a magical cure would be offered if I just became a little bit sicker? Ultimately, it wasn't magic that helped, but time-and the willingness of the few who patiently sat with me while I weathered the agony of very slowly getting better. It can be excruciating to bear witness to someone's pain when we have no solutions, and yet, the power of that sharing of suffering is often what is needed most on the darkest of days.

We know the statistics on burnout, anxiety, and depression. Our residents engage in emotionally draining work while sleep deprived and attempting to juggle home life. We design wellness initiatives and we make accommodations for residents to attend medical and mental health appointments. Are they going? How many are silently suffering, artfully hiding their distress, seemingly thriving? How do we reach them? How do we make it okay not to be okay? One thing we can do from positions of leadership is to demonstrate vulnerability, to share our struggles openly. It is then that we begin to break down barriers to care that are entrenched among physicians, a curriculum hidden so deeply that it is hard to reach. My story isn't extraordinary by any stretch. As Michael Rose concluded in a moving essay about his experience as a patient and medical student, "The key to overcoming the status quo goes beyond institutions and policy. It lies with individuals and our willingness to share personal 


\section{ON TEACHING}

stories-and not a few, but many." I I am one of the many-are you?

\section{References}

1. Rose MR. SIGECAPS, SSRIs, and silence-life as a depressed med student. N Engl J Med.

2018;378(12):1081-1083. doi:10.1056/

NEJMp1716893.

Shannon E. Scott-Vernaglia, MD, is Pediatrics Residency Program Director, MassGeneral Hospital for Children, and Assistant Professor, Harvard Medical School.

Corresponding author: Shannon E. Scott-Vernaglia, MD,

MassGeneral Hospital for Children, CPZS-588, 175 Cambridge Street, Boston, MA 02114, 617.726.2687, sscottvernaglia@mgh.harvard.edu 